\title{
Experiences from Transforming a Lecture "Communication Systems" from Presence to Virtual Format during the COVID-19 Pandemic
}

\section{Thomas Fuhrmann}

Faculty for Electrical Engineering and Information Technology, OTH Regensburg, Germany.

\begin{abstract}
This article describes the transition of an existing lecture "Communication Systems" from a conventional presence format to a virtual format due to the COVID-19 pandemic. The process of transformation and the evolution of the lecture during the virtual semester are characterized. The changed exam format from presence to virtual format with the experiences are described. Results of a student evaluation at the end of the semester are shown. The lessons learned are summarized also for the time after the COVID-19 pandemic.
\end{abstract}

Keywords: Communication engineering; electrical engineering education; STEM; computer aided instruction; electronic learning. 


\section{Introduction}

The year 2020 was a big challenge for universites around the world to rapidly switch teaching from presence to online formats (Jesionkowska, Fominykh, Wild, \& Molka-Danielsen, 2020; Mishra, Gupta, \& Shree, 2020; Mohmmed, Khidhir, Nazeer, \& Vijayan, 2020). OTH Regensburg reacted to the COIVD-19 pandemic with the following measures:

- Postponed semester start from 15 March to 20 April 2020.

- Complete closure of university buildings for students.

- Virtualization of lectures, seminars and lab courses as far as possible.

The transformation of the course "Communication Systems" in the summer semester 2020 during the pandemic is described. In the next Section, the course structure is explained. Section 3 describes the improvised lectures changes due to the lockdown. The seen changes in didactics are explained in Section 4 and the changes of the integrated practical part are described in Section 5. Also the exam was virtualized, see Section 6. Feedback from students is shown in Section 7 and Section 8 lists the lessons learned from this semester.

\section{Course Structure}

This course is a technical elective course in the sixth semester within the "Bachelor of Electrical Engineering" program. It is given during summer semesters with four lecture hours per week for 15 weeks with a nominal workload of 5 ECTS Credits. A moodle platform is used for providing course material, information and other course related support to the students. The course script is a PDF file with extensively written course material including texts, tables, graphs and pictures. The course is structured with the main topics:

- Principles of communication systems, communication media and multiplexing.

- ISO-OSI layer model, TCP/IP stack, network topologies and access methods.

- Coding, error detection, error correction and cryptography.

- Analogue and digital modulation formats.

- Introduction to optical communication systems and Digital Subscriber Line (DSL).

About one quarter of the course time is reserved for practical lessons in the lab. The goal of these practical lessons is that students get hands-on experience when dealing with a small engineering project task. Group work in presence in the lab is seen to be important for gaining technical and social skills.

\section{Course Start}

The semester start was postponed to 20 April 2020. Courses could start on 16 March 2020 on a voluntary basis for students. Those who were not able to follow the courses during the 
hard lockdown in Germany should have a repetition phase at the beginning of the regular lecture time in April. The optional lecture start was organized as a self-study phase. The script was provided via the moodle platform. To compensate for the missing personal interaction, the following additional materials were provided:

- A weekly learning plan with the script chapters that should be read.

- Learning goals for all chapters were formulated.

- Links to additional online resources.

- Questions to test students' knowledge.

A regular chat session once per week and a discussion forum on moodle were introduced.

About 15 students started the course and also finished it. Most students used the provided resources for self-learning. Several questions were asked and intense discussions happened during this phase. Regular lectures started after the four first weeks (plus one week Easter holidays) with a repetition phase of the first script chapters. All regular lectures were done with live streams using a video communication platform. Videos were recorded and stored for students who were not able to participate during lecture hours.

\section{Evolution of Didactics During Virtual Lectures}

The general didactic concept of this course given in presence is strongly dialogue oriented. The script is not the main part of this course, the script contains mainly additional material for the students to read at home and deepen their knowledge. Presence time is used to explain the topics, to discuss with the lecturer and in student groups, and to solve questions and problems. This presence lecture concept with a lot of personal interaction was very difficult to transfer to the virtual lecture format. Some adjustments to the lecture format were made due to the lack of personal interaction. Before this Summer Semester 2020, there was only a very limited virtual lecture experience by the students and by the lecturer. All participants had to learn from scratch how to do virtual lectures.

Table 1 shows statistical data about the lecture structure during the semester. The left column shows the lecture week and the second column the number of recorded videos. In the third column, the average length of each video and the fourth column the longest video is shown. The number of video views can be seen in the second right column and the number of group work phases in the right column. It is known that the student concentration drops steadily during lectures (Stuart \& Rutherford, 1978). Regular breaks should be therefore made for a short regeneration phase. The lengths of speaking blocks were therefore limited to about $20 \ldots 30$ minutes. Between these speaking blocks, questions or small challenges were given to be discussed within random groups of $2 \ldots 3$ students. During these group works, the lecturer 
visited the groups, asked if everything is understood and discussed questions with the students.

Table 1. Analysis of Virtual Lectures.

\begin{tabular}{cccccc}
\hline Week & Videos & Av. Video Length & Max Video Length & Video Views & Group Works \\
\hline $1 \ldots 4$ & 0 & & Optional Self-Learning Phase & \\
5 & 4 & $12: 48$ & $23: 07$ & 80 & 2 \\
6 & 6 & $12: 14$ & $21: 37$ & 71 & 2 \\
7 & 3 & $33: 22$ & $39: 38$ & 32 & 2 \\
8 & 6 & $16: 30$ & $34: 32$ & 73 & 4 \\
9 & 4 & $17: 11$ & $30: 22$ & 45 & 4 \\
10 & 5 & $11: 05$ & $22: 16$ & 39 & 4 \\
11 & 5 & $18: 31$ & $32: 19$ & 19 & 3 \\
12 & 4 & $20: 21$ & $31: 55$ & 15 & 2 \\
13 & 3 & $22: 13$ & $32: 53$ & 9 & 2 \\
14 & 2 & $19: 12$ & $20: 42$ & 3 & 2 \\
15 & 1 & $39: 25$ & $39: 25$ & 2 & 16 \\
16 & 3 & $14: 56$ & $31: 05$ & Questions \& Answers & \\
17 & 0 & & & & 2 \\
\hline & & & & 2 & \\
\hline
\end{tabular}

\section{Practical Experiments}

In the years before, a practical part was carried out in the lab with the following tasks:

- A telephone communication cable with unknown parameters was analyzed.

- A simple optical communication system was built by the students.

These practical tasks were not possible due to the COVID-19 pandemic. It was therefore decided to do a practical part using a simulation software. After evaluation features and complexity of different scientific software packages for simulating communication systems, GNU Radio was chosen (https://www.gnuradio.org/). This is an open source software to simulate communication systems. A large community exists for providing model solutions and discussing about questions. Simulations were mainly done in weeks 13...16 (see Table 1). Some easy examples for communication system simulations were given by the lecturer. The students should change simulation parameters, see what happens and explain the reasons. 
They should also modify the simulated communication systems according to the topics in the lecture. This practical part worked for some students very well, other students had diverse problems with the software.

\section{Exam Concept}

Before COVID-19, the exam was a presence exam with about 15 questions and a time limit of 90 minutes. A non-programmable calculator and four hand written pages were allowed. This type of exam was not possible due to the pandemic. The lecturer decided to change this exam to a case study that can be worked out at home. Students had to declare the will to participate in the exam. They got the exam task simultaneously by email and had to send the solution by a certain date and time back. During the exam time, a forum was open at the moodle course where students could ask and answers were posted for all students. In this case study, a scenario was given to design a fast internet connection solution for a rural village with detailed parameters. Students had two weeks time to write about ten pages with their chosen solution. The grading was done by giving points for formal correctness, the completeness and plausibility of the solution.

\section{Feedback from Students}

The course was evaluated using a web-based survey tool. Students' overall satisfaction with the course was high. The most important results are shown below.

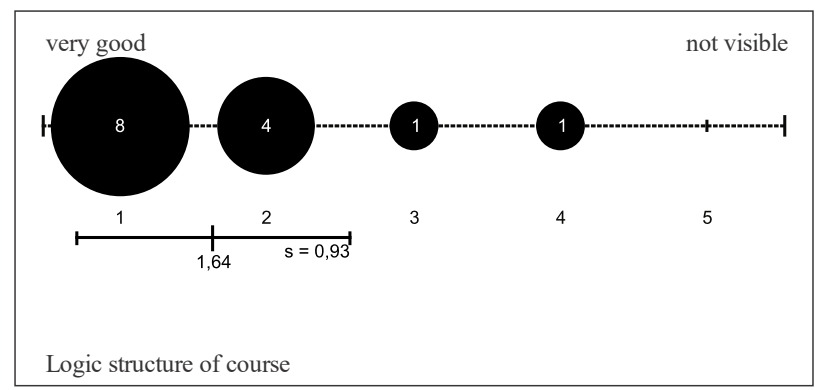

Figure 1. Logic structure of course.

The logic structure of the course is seen as good to very good (Figure 1). Compared to surveys from the presence courses, no loss in the course structure is seen. This very valuable feedback leads to the conclusion that the overall course transition was successful. Students saw a high knowledge gain during the course (Figure 2). This leads to the conclusion that no severe deficits are in the virtual course. All essential topics could be explained with a similar success using video lectures compared to presence lectures. 


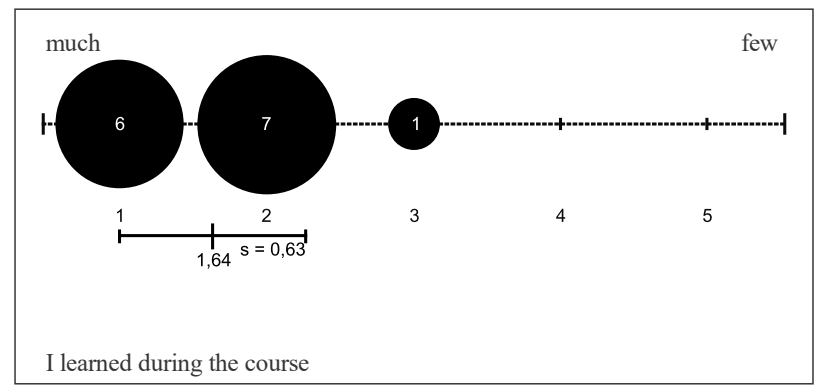

Figure 2. Knowledge gain during the course.

The question of knowledge gain is closely connected to the satisfaction with the virtual teaching methods that are shown in Figure 3. Students evaluated that appropriate virtual methods were used and evaluated these as good to very good.

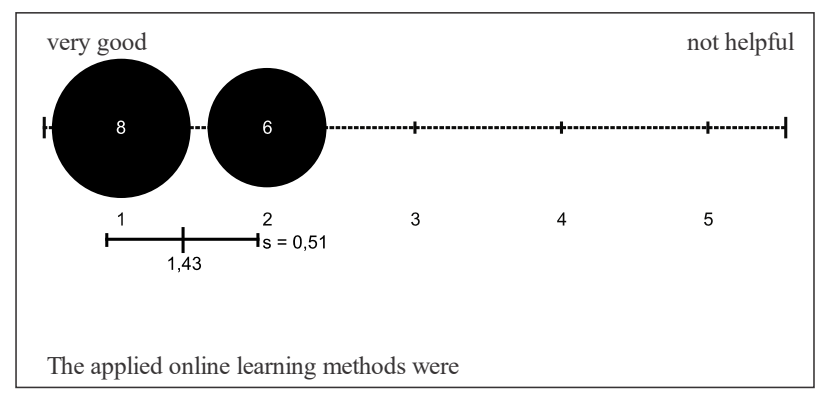

Figure 3. Usage of online learning methods.

Figure 4 shows the satisfaction with the communication media that were used by the lecturer during the online semester. It shows that the video lectures, the script on the moodle platform and email for discussion were appropriate. A personal feedback said that especially the short video talks together with many group works were seen as very positive.

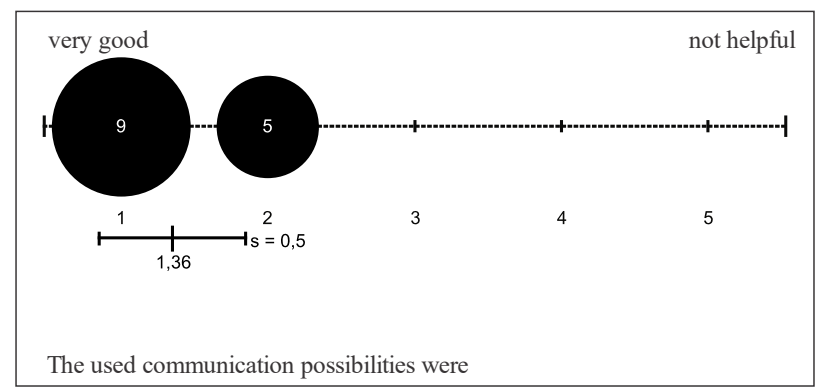

Figure 4. Communication media during online lectures.

It is very important that the recordings were very useful, as shown in Figure 5. Early recordings were viewed much more often than late recordings for an unknown reason. 


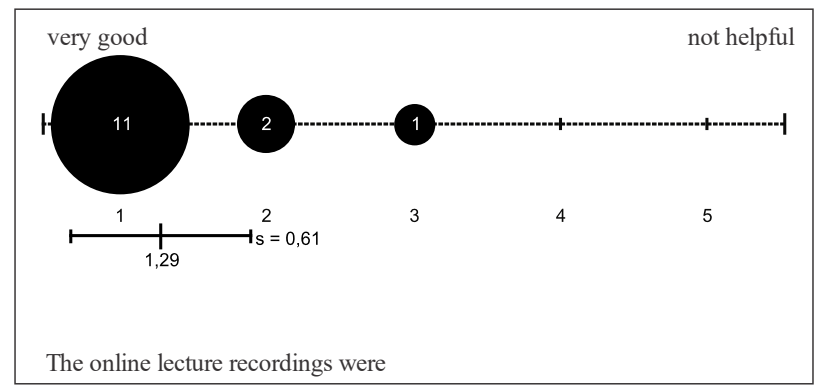

Figure 5. Value of lecture recordings.

Students state that the contact possibilities with the lecturer were absolutely sufficient, as Figure 6 shows. It can be concluded that email contact, a moodle forum and chats during video lectures are sufficient, additional chat possibilities are not necessary.

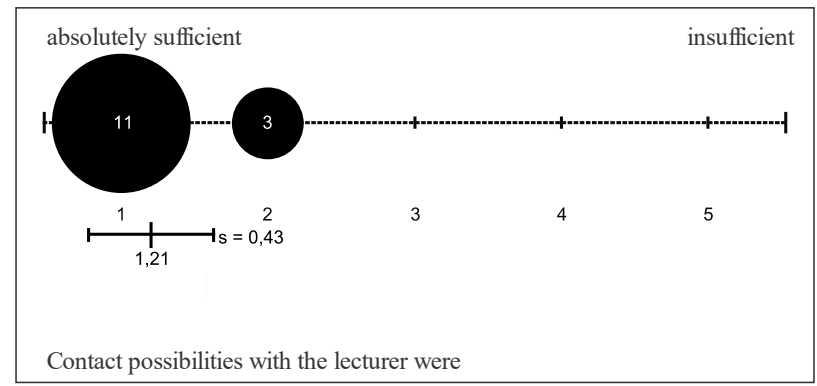

Figure 6. Contact possibilities with lecturer.

Positive comments were mainly about the group sessions where students had the possibility to discuss about topics and questions. The students wanted to have a more detailed concept to use this GNU Radio. This deficit was also seen by the lecturer. Student showed overall understanding for the difficult situation and for the efforts to do a virtual course. Summarized, students were overall satisfied with the virtual course. From this basis, it is planned to develop the course further during the next years.

\section{Lessons Learned}

Lessons learned from virtual lectures:

- It was some work but no challenge to modify the course for the virtual lecture.

- The main challenge during the lectures was that all student switched off their cameras. It was unclear if students were engaged and understood the topics due to missing visual feedback. 
- Discussions were much more difficult due to the missing personal interaction. Most students did not dare to ask questions or discuss in the big group. Most intense discussions happened when splitting the course to smaller groups for special topics.

- Group works were not easy but possible by using screen sharing. Students needed some weeks to get accustomed to virtual group works. After this first phase, virtual collaboration was nearly as effective as collaboration in presence.

- It was very difficult for the lecturer to recognise if students are still participating in the lecture or if they are already lost.

Lessons learned from the practical part of the course:

- Students had no chance to work in groups in the lab to develop own systems. This hands-on experience could not fully be replaced by simulations. Essential competencies like project work in teams could not be taught.

- Students needed more guidance than expected for simulations. A tutorial with structured tasks is very helpful to have a starting point for developing own simulations. It is preferrable to give simulation tasks parallel to the corresponding lecture chapters.

Lessons learned for the time after COVID-19:

- Personal contact is essential to get feedback about the learning success. If no faceto-face contact is possible, appropriate alternatives have to be established.

- Lab projects in groups to get hands-on experience is essential for students to learn engineering working principles. Simulations can give additional competencies but can't fully replace hands-on experience in the lab.

- The lecturer had to leave the standard didactic program. New didactic interactions were tested in virtual sessions. The lecturer broadened the didactic portfolio also with more opportunities for future semesters.

\section{References}

Jesionkowska, J., Fominykh, M., Wild, F., \& Molka-Danielsen, J. (2020). Pandemic-Induced Constraints on Rapid Transformation to Digital Education. CEUR Workshop Proceedings. Retrieved fom http://ceur-ws.org/Vol-2676/paper3.pdf.

Mishra, L., Gupta, T., \& Shree, A. (2020). Online teaching-learning in higher education during lockdown period of COVID-19 pandemic. International Journal of Educational Research Open, 1, 100012.

Mohmmed, A. O., Khidhir, B. A., Nazeer, A., \& Vijayan, V. J. (2020). Emergency remote teaching during Coronavirus pandemic: the current trend and future directive at Middle East College Oman. Innovative Infrastructure Solutions, 5(3), 1-11. 
Stuart, J., \& Rutherford, R. D. (1978). Medical student concentration during lectures. The lancet, 312(8088), 514-516. 